\title{
Signature of dynamical heterogeneity in spatial correlations of particle displacement and its temporal evolution in supercooled liquids
}

\author{
Indrajit Tah $\odot^{*}$ and Smarajit Karmakar $\odot^{\dagger}$ \\ Tata Institute of Fundamental Research, 36/P, Gopanpally Village, Serilingampally Mandal, Ranga Reddy District, Hyderabad 500107, India
}

(Received 18 July 2019; accepted 4 June 2020; published 25 June 2020)

\begin{abstract}
The existence of heterogeneity in the dynamics of supercooled liquids is believed to be one of the hallmarks of the glass transition. Intense research has been carried out in the past to understand the origin of this heterogeneity in dynamics and a possible length scale associated with it. We have done extensive molecular dynamics simulations of a few model glass-forming liquids in three dimensions to understand the temporal evolution of the dynamic heterogeneity and the heterogeneity length scale. We find that although the strength of the dynamic heterogeneity is maximum at a timescale close to the characteristic $\alpha$-relaxation time of the system, dynamic heterogeneity itself is well developed at a timescale as short as the $\beta$-relaxation time and survives up to a timescale as long as a few tens of the $\alpha$-relaxation time. Moreover, we discovered that the temperature dependence of the heterogeneity length remains the same in the whole time window, in agreement with the inhomogeneous mode-coupling theory, although its absolute value changes over time in a nonmonotonic manner.
\end{abstract}

DOI: 10.1103/PhysRevResearch.2.022067

Dynamic heterogeneity $(\mathrm{DH})$ is ubiquitous in a vast variety of natural processes spanning from molecular systems to biological cells and tissues [1-8]. The existence, characterization, and its role in different dynamical processes, particularly in the dynamics of glass-forming liquids approaching the glass transition, is an active field of research [9-17]. Extensive studies $[9,11,18-20]$ have been performed in the past to understand the behavior of $\mathrm{DH}$ at the characteristic long relaxation timescale or the $\alpha$-relaxation timescale $\tau_{\alpha}$ (defined later) [21], and only a handful studies have been done at the shorter $\beta$ relaxation timescale [22]. In Ref. [22], it has been shown that the $\beta$-relaxation time $\tau_{\beta}$ (defined later) has a strong finite-size effect, which can be rationalized if one assumes the existence of a growing correlation length. It was surprisingly found in Ref. [22] that the temperature dependence of this growing correlation length at the $\beta$-relaxation time is the same as that of the heterogeneity length scale obtained at the $\alpha$-relaxation time. This observation is very surprising as these timescales can differ by many orders of magnitude, especially at low temperatures.

The main goals of this Rapid Communication are twofold. The first goal is to find direct signatures of DH in the dynamics at $\tau_{\beta}$. Then we would like to understand the subsequent growth and temporal evolution of $\mathrm{DH}$ at timescales ranging from $\tau_{\beta}$ to at least an order of magnitude larger than $\tau_{\alpha}$. As most of the research works have focused on the characteriza-

\footnotetext{
*indrajittah@tifrh.res.in

†smarajit@tifrh.res.in
}

Published by the American Physical Society under the terms of the Creative Commons Attribution 4.0 International license. Further distribution of this work must maintain attribution to the author(s) and the published article's title, journal citation, and DOI. tion of DH in the $\alpha$-relaxation timescale, it is very important to comprehend the time evolution of $\mathrm{DH}$ in the intermediate as well as long timescale compare to $\tau_{\alpha}$, to understand the role of DH in the glass transition. The main results of this Rapid Communication are the observation of the signature of $\mathrm{DH}$ in the displacement fields of particles at $\tau_{\beta}$ and the survival of DH at timescales that are larger than $\tau_{\alpha}$ by at least a factor of 10 in the studied temperature range. We have also discovered that the temperature dependence of the heterogeneity length scale $\xi$ remains the same throughout the studied time window, but the region of heterogeneity or the spatial extent of heterogeneity changes with time in a nonmonotonic way with the maximum appearing at or near $\tau_{\alpha}$.

Although in Ref. [22] it was shown that DH seems to be quite well developed in the system at $\tau_{\beta}$, it was not immediately clear how particle motions at this short timescale get affected due to the presence of the heterogeneity. In other words, it is not immediately clear whether particle motions at $\tau_{\beta}$ are correlated over $\xi$. In this study, $\tau_{\beta}$ is defined from the logarithmic derivative of mean squared displacement (MSD) with time [22] (see Ref. [23] for further details).

To measure the spatial correlation and to extract the associated length scale in the displacements of particles at $\tau_{\beta}$, we have implemented the procedure given in Refs. [24-27]. Note that this measure of the spatially correlated motion in supercooled liquids does not depend on arbitrary cutoff parameters as already conclusively shown in Refs. [24-26] for DH at $\tau_{\alpha}$. The spatial correlation of the particle displacements $g_{\text {uu }}(r, \Delta t)$ is defined as

$$
g_{u u}(r, \Delta t)=\frac{\left\langle\sum_{i, j=1, j \neq i}^{N} \mathbf{u}_{i}(t, \Delta t) \mathbf{u}_{j}(t, \Delta t) \delta\left(r-\left|\mathbf{r}_{i j}(t)\right|\right)\right\rangle}{4 \pi r^{2} \Delta r N \rho\left\langle u^{2}(\Delta t)\right\rangle},
$$



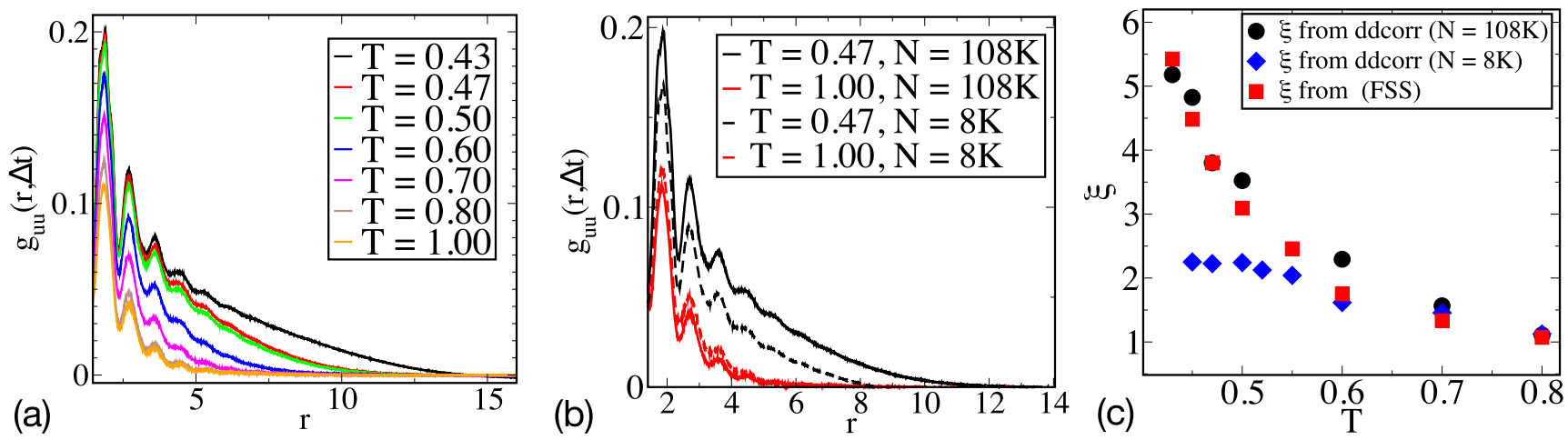

FIG. 1. (a) Displacement-displacement correlation $g_{\text {ии }}(r, \Delta t)$ at $\Delta t=\tau_{\beta}$ for $3 \mathrm{dKA}(N=108000)$. (b) System size dependence of $g_{\text {uи }}(r, \Delta t)$ for the 3dKA model. (c) The dynamic length scale as a function of time and compared with the corresponding quantities obtained using conventional finite-size scaling (FSS) at $\tau_{\beta}$.

where $\mathbf{u}_{i}(t, \Delta t)=\mathbf{r}_{i}(t+\Delta t)-\mathbf{r}_{i}(t)$ is the vector displacement of the particle between time $t$ and $t+\Delta t .\left\langle u^{2}(\Delta t)\right\rangle=$ $\left\langle\frac{1}{N} \sum_{i=1}^{N} \mathbf{u}_{i}(t, \Delta t) \cdot \mathbf{u}_{i}(t, \Delta t)\right\rangle . \quad \mathbf{r}_{i j}(t)=\mathbf{r}_{j}(t)-\mathbf{r}_{i}(t)$ is the distance between the $i$ th and $j$ th particles. Note that our definition of the displacement-displacement correlation is slightly different from the definition given in Ref. [25]. In Ref. [25], the scalar displacement-displacement $\left[u_{i}(t, \Delta t)=\right.$ $\left.\left|\mathbf{u}_{i}(t, \Delta t)\right|\right]$ correlation $g_{u u}^{s}(r, \Delta t)$ (see Ref. [23]) is considered, whereas we have considered the vector displacement of the particles [28]. It captures the orientational as well as translational correlation in the particle displacements within the time of observation.

We have performed extensive computer simulations of four well-studied model glass formers in three dimensions with different interparticle potentials over a wide range of temperatures. The model systems studied are the following: (i) $3 \mathrm{dKA}$ [29], (ii) $3 \mathrm{dR} 10$ [30], (iii) 3dIPL [31], and (iv) $3 \mathrm{dHP}[32,33]$. The details of the models and simulations are given in Ref. [23]. We find that the growth of DH identified using $g_{\text {uu }}(r, \Delta t)$ shows a strong system size dependence at least at $\tau_{\beta}$. This was not the case in Refs. $[34,35]$ when the correlation function was computed at $\tau_{\alpha}$. Thus we have computed $g_{u u}(r, \Delta t)$ for different system sizes $N=8000$, $N=10000$, and $N=108000$.

In Fig. 1(a) we show the $g_{\text {uu }}(r, \Delta t)$ for $N=108000$. It is observed that $g_{u u}(r, \Delta t)$ exhibits damped oscillation which is in agreement with previous numerical [26,36] as well as experimental studies [37]. The correlation function decays to zero exponentially as a function of distance $r$, and with decreasing temperature, the dynamics of the liquid becomes more heterogeneous as the correlation between the particles' displacements in space extends up to a larger distance as shown in the Fig. 1(a). It physically means that particles in the liquids are moving in a cooperative fashion with a monotonically increasing size of the cooperative region as the temperature is lowered. We find a strong system size dependence in $g_{u u}(r, \Delta t)$ as shown in Fig. 1(b). We have computed the correlation for $N=108000$ and $N=8000$ for the 3dKA model [see Fig. 1(b)] to show that at low temperature, the relative correlation increases with an increase in system size. For the robustness of our results, we have calculated the correlation for the other two model systems. We found the results are quantitatively similar (see Ref. [23]).
In Fig. 1(c), we show the temperature dependence of the heterogeneity length scale for the 3dKA model. As expected, strong finite-size effects are observed in the obtained $\xi$ as well. $\xi$ 's obtained from a very large system size $(N=108000)$ grow very similarly to the dynamical length scale obtained from the finite-size scaling (FSS) of $\tau_{\beta}$ [22]. For the $N=8000$ system size one observes that $\xi$ grows mildly, thus studies on smaller system sizes would have led to a conclusion that $\mathrm{DH}$ is not very strong at the $\beta$-relaxation time. We now focus on the temporal evolution of $\mathrm{DH}$ and $\xi$ across the whole range of timescales that can be accessed in simulation.

Equipped with the method of block analysis, introduced in Ref. [38], a systematic study of the temperature dependence of the dynamical length scale across different timescales for different model glass-forming liquids became computationally feasible. In Ref. [35], although DH was studied over different timescales, a systematic study on the temperature dependence of $\xi$ was not done. Usually, four-point correlation functions $g_{4}(r, t)$ and the corresponding susceptibilities $\chi_{4}(t)$ [39] are used to study DH. $\chi_{4}(t)$ is related to fluctuations in the two-point function $Q(t)$. The Fourier transform of $g_{4}(r, t)$ is known as the four-point structure factor $S_{4}(q, t)$ and related to $\chi_{4}(t)$ as $\lim _{q \rightarrow 0} S_{4}(q, t) \equiv \chi_{0}(t)$. $\tau_{\alpha}$ is defined as $\left\langle Q\left(t=\tau_{\alpha}\right)\right\rangle=1 / e$, where $\langle\cdots\rangle$ denotes ensemble averages (see Ref. [23] for further details and definitions).

To perform the block analysis, we equilibrate a large system of $N=108000$ particles and measure various quantities of interest by coarse-graining over different block sizes $L_{B}$. We then obtain the dynamic length scale $\xi$ by a FSS analysis of $\chi_{4}\left(L_{B}, t\right)$. In previous studies $[38,40]$, the dependence of $\chi_{4}^{P}\left(L_{B}\right)$, the maximum intensity (peak value) of dynamical susceptibility on the block size, was studied and the dynamic heterogeneity correlation length $\xi$ has been estimated by a FSS analysis using the following scaling form,

$$
\chi_{4}^{P}\left(L_{B}, T\right)=\chi_{4}^{P}(\infty, T) f\left[L_{B} / \xi(T)\right],
$$

where $\chi_{4}^{P}(\infty, T)$ is the $L_{B} \rightarrow \infty$ value of dynamical susceptibility at a temperature $T$. In this work we have done a similar scaling analysis for the block size dependence of the intensity of the dynamical heterogeneity at a few particular timescales $t=\tau_{\alpha} / 3, \tau_{\alpha} / 2, \tau_{\alpha}, 3 \tau_{\alpha}$.

In Fig. 2 we have plotted the results for the 3dKA model system. In the top panels, we reported the block size de- 


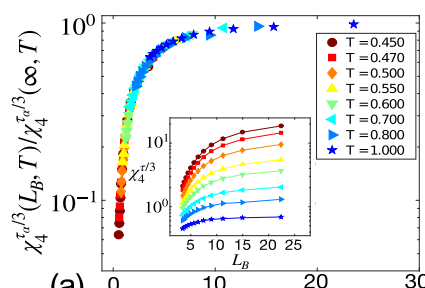

(a) ${ }^{10} / \xi(T)$
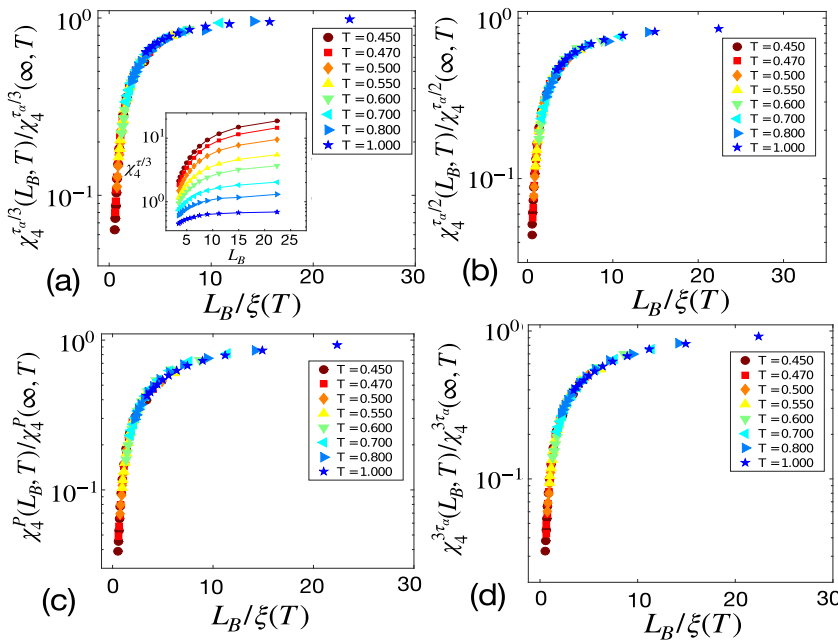

FIG. 2. Block size dependence of $\chi_{4}$ at different time intervals. The collapse of data is done by rescaling the $x$ axis to get the length scale $\xi(T)$. The inset shows the unscaled data. Figures are shown at different time intervals: (a) $\tau_{\alpha} / 3$, (b) $\tau_{\alpha} / 2$, (c) $\tau_{\alpha}$, and (d) $3 \tau_{\alpha}$, respectively.

pendence of $\chi_{4}\left(L_{B}, T\right)$ (inset) and the scaling collapse of $\chi_{4}\left(L_{B}, T\right)$ at $\tau_{\alpha} / 3$ [Fig. 2(a)] and $\tau_{\alpha} / 2$ [Fig. 2(b)] timescales, respectively. A similar analysis also is shown in the bottom panels at timescales $\tau_{\alpha}$ [Fig. 2(c)] and $3 \tau_{\alpha}$ [Fig. 2(d)]. The scaling observed in all these four cases is indeed very good and the calculated length scales from the FSS analysis of the block method are found to be in good agreement with the $\xi$ obtained from the wave-vector dependence of $S_{4}(q, t)$ [41] (discussed below). In FSS, $\xi(T)$ is known up to a unique multiplicative factor for all temperatures. In order to fix this uncertainty, $\xi$ obtain from FSS is scaled to match with $\xi$ obtained from $S_{4}(q, t)$ at one temperature.

By fitting the $q$ dependence of $S_{4}(q, t)$ for small $q$ values to the Ornstein-Zernike $(\mathrm{OZ})$ form $S_{4}(q, t) \simeq \chi_{0}(t) /\left[1+(q \xi)^{2}\right]$, one can also obtain $\xi$. It has already been shown that the heterogeneity length scale obtained from FSS of the block method is in good agreement with the same obtained from $S_{4}\left(q, t=\tau_{\alpha}\right)$. In the top panels of Fig. 3 we plot the wavevector dependence of the inverse of four-point structure factor $S_{4}(q, t)$ for the $3 \mathrm{dKA}$ model for two different times, $\tau_{\alpha} / 3$ [Fig. 3(a)] and $3 \tau_{\alpha}$ [Fig. 3(b)]. One can clearly see that the $\mathrm{OZ}$ form fits the data very well, thus the extracted length scale will be quite accurate. In the bottom panels of the same figure, the temperature dependences of the length scales computed by different methods are compared for $t=\tau_{\alpha} / 3$ [Fig. 3(c)] and $t=3 \tau_{\alpha}$ [Fig. 3(d)]. The legend " $\xi$ from FSS" refers to the length scale obtained from the finite-size scaling at $t=\tau_{\beta}$ and taken from Ref. [40]. Note that $\xi$ 's from FSS are scaled at $T=0.80$. It is worth highlighting that these results suggest that the temperature dependence of $\xi$ is the same across timescales starting from $\tau_{\beta}$ to at least $3 \tau_{\alpha}$. To check the robustness of our results, the length scales for the other two models (3dR10 and 3dIPL) are also computed and the temperature dependences of the length scales are found to be the same over the time interval $\left(\tau_{\beta}, 3 \tau_{\alpha}\right)$ (see Ref. [23] for details).
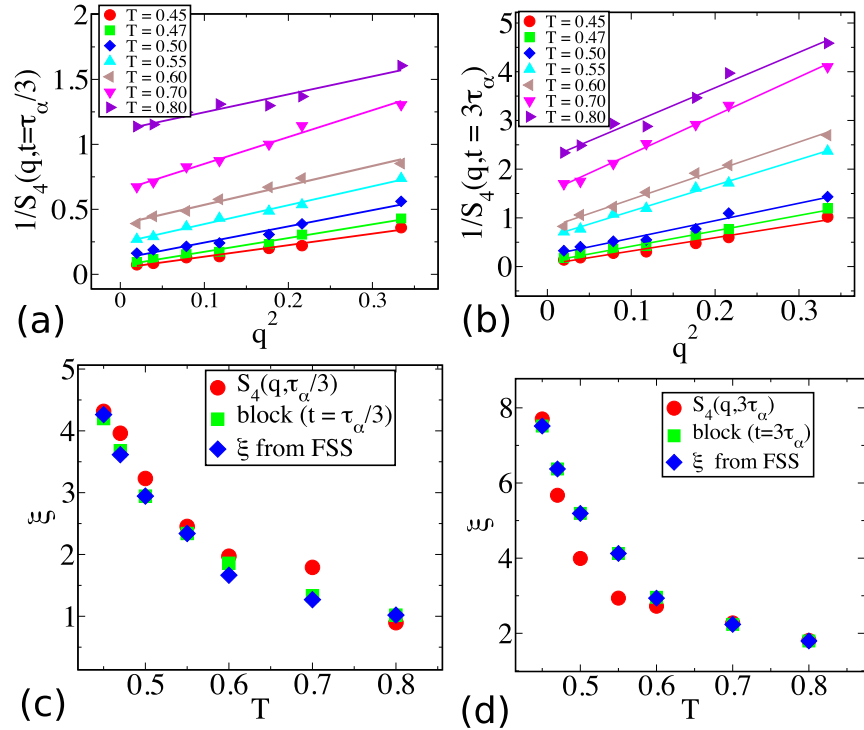

FIG. 3. (a) We plot $1 / S_{4}(q, t)$ vs $q^{2}$ for the 3dKA model at $t=$ $\tau_{\alpha} / 3$ and (b) $t=3 \tau_{\alpha}$ and we get the dynamical length scale by fitting $S_{4}(q, t)$ to the Ornstein-Zernicke (OZ) form $S_{4}(q, t)=\frac{S_{4}(q \rightarrow 0, t)}{1+(q \xi)^{2}}$ in the range $0<q<0.578083$. (c) and (d) Comparison of different length scales obtained by using the conventional finite-size-scaling (FSS) method of $\chi_{4}^{P}[40]$ and block analysis method.

Our results are in reasonable agreement with the inhomogeneous mode-coupling theory (IMCT) prediction [42], which says that there exists a unique growing dynamical correlation length that grows as $\xi \sim\left|T-T_{d}\right|^{-v}$ with a critical exponent $v=1 / 4$, where $T_{d}$ is the MCT divergence temperature. IMCT also predicts that this length scale remains the same in both the $\alpha$ - and $\beta$-relaxation regimes. We also found that $v \simeq 1 / 4$ is not inconsistent with our low-temperature data for all the model systems (see Ref. [23]). Although the temperature dependence of $\xi$ remains the same over the studied timescales for all model systems, the spatial extent of the heterogeneity is observed to increase up to a particular timescale and then starts to decrease.

Next, we examine the power-law relation between $\chi_{4}(T)$ and $\xi(T)$. According to IMCT [42-44], one expects a power relation between $\chi_{3}\left(\tau_{\alpha}\right)$ (a three-point correlator which is similar to $\chi_{4}$ at least in the scaling behavior) and $\xi\left(\tau_{\alpha}\right)$ as $\chi_{3}\left(\tau_{\alpha}\right)=\xi^{2-\eta}\left(\tau_{\alpha}\right)$ with the theoretically predicted exponent $2-\eta=4$ in the $\alpha$ regime and 2 for the $\beta$ regime [45]. Following Ref. [38] we have done the scaling analysis of $\chi_{4}^{t^{*}}(T)$ to obtain the exponent $2-\eta$ at different times $t^{*}$. In the large system size limit, $L_{B} \gg \xi$, the $L_{B}$ dependence should disappear in the scaling relation in Eq. (2) and it should approach a constant value for $x \gg 1$. On the other hand, for $\xi \rightarrow \infty$ and $L_{B}$ remaining finite the dependence of $\chi_{4}$ on $\xi$ should go away. This implies that the scaling function $f(x)$ should be proportional to $x^{2-\eta}$ at $x \rightarrow 0$ and $\chi_{4}^{t}\left(L_{B}, T\right)$ should grow as $L_{B}^{2-\eta}$. We show the results of such an analysis for the 3dKA model in Fig. 4. The exponent value is found to be $\eta \simeq 0$ for both $t=\tau_{\alpha} / 3$ as well as at $3 \tau_{\alpha}$. This is different from the exponent $(2-\eta \simeq 4)$ predicted by IMCT in the $\alpha$ regime but is in very good agreement with the prediction at the $\beta$ regime. This observation can 


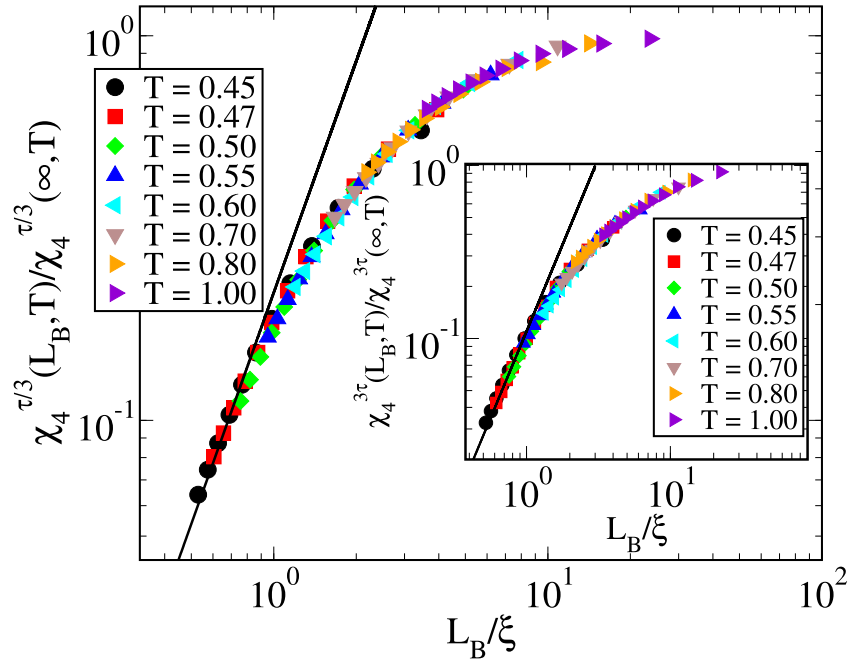

FIG. 4. Scaling analysis for the $3 \mathrm{dKA}$ model to obtain the exponent $\eta$ at time intervals $t=\tau_{\alpha} / 3$ and $t=3 \tau_{\alpha}$ (inset). The black solid line represents the function $f(x)=x^{2-\eta}$, where $2-\eta=2$, thus $\eta=0$.

be rationalized if one assumes that the amount of activated relaxation at a short timescale will be less compared to the $\alpha$-relaxation timescale and the IMCT approximation will then become reasonable. Thus we can expect to have a reasonable agreement with IMCT predictions at short timescales but not as good at longer timescales. Our results very nicely highlight this agreement with good quality data. We have done a similar analysis for other model systems (3dIPL and $3 \mathrm{dR} 10$ ) and found that the exponent $\eta \simeq 0$ (see Ref. [23] for details).

Next, we look at the time evolution of the DH length scale. In Ref. [41], it was shown that the time dependence of $\xi(t)$ is the same as $\chi_{4}(t)$, which is in contradiction with the results reported in Ref. [46]. In Ref. [46], $\xi$ is found to increase monotonically with time, in partial agreement with the results reported in Ref. [47] for hard-sphere systems. Moreover, in Ref. [47] it is found that $\xi$ saturates to a plateau at a later time. On the other hand, IMCT predicts $\xi$ remains the same in between the $\tau_{\beta}$ and $\tau_{\alpha}$ timescale. We then look at the mutual time evolution of $\xi$ and $\chi_{4}$ for all the model systems to understand the contradiction in the reported results in the literature.

In Fig. 5(a), the time dependence of $\xi(t)$ for the $3 \mathrm{dR} 10$ model is shown. It is clear that $\xi(t)$ grows up to $\tau_{\alpha}$ and decreases at a later time, in agreement with Ref. [41]. We also found a completely different behavior of $\xi(t)$, if we compute it from $g_{u u}(r, t)$. $\xi(t)$ seems to remain the same in magnitude throughout the entire time window from $\tau_{\beta}$ to $\tau_{\alpha}$ [magenta $\nabla$ in Fig. 5(a)]. We found a similar length scale dependence with time for the 3dKA model as well. (See Ref. [23].) Next, we look at the results obtained for the 3dHP model (see Ref. [23]) [32], a paradigmatic model in the context of jamming physics. For the 3dHP model, $\xi(t)$ shows a peak at a timescale close to $4 \tau_{\alpha}$ [Fig. 5(b)] for the softness parameter of the potential $\delta=2$ (see Ref. [23] for a definition of $\delta$ ). This implies that $\xi(t)$ increases even though the overall strength of the heterogeneity decreases after $\tau_{\alpha}$. To study the
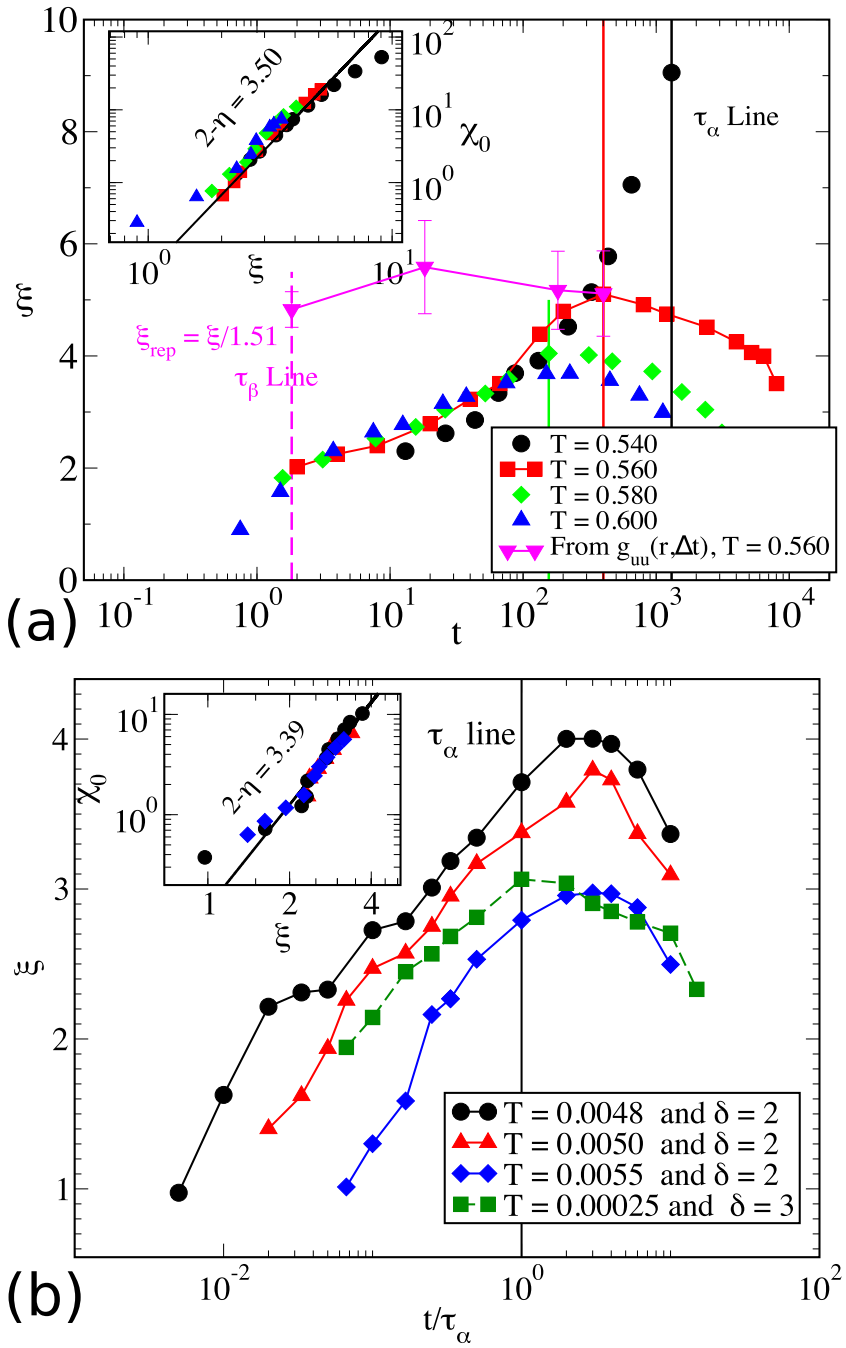

FIG. 5. (a) The dynamic length scale vs time (semilog plot) for the $3 \mathrm{dR} 10$ model. Vertical solid lines represent the $\tau_{\alpha}$ line at different temperatures. The vertical dotted line is the $\tau_{\beta}$ line for $T=0.560$. In the inset we show the power-law dependence of $\chi_{0}(t)$ vs $\xi(t)$. $\xi_{\text {rep }}$ (magenta $\nabla$ ) represents the length obtained from $g_{\text {uи }}(r, t)$. (b) Similar plot for the 3dHP model. In the $x$ axis, time is rescaled by its corresponding $\tau_{\alpha}$ value. The vertical line represents the $\tau_{\alpha}$ line for the $3 \mathrm{dHP}$ model.

effect of density $(\rho)$ on the temporal evolution of $\xi(t)$, we have done a simulation at different densities $\rho=0.81$ and temperature $T=0.0048$ for the $3 \mathrm{dHP}$ model. The results are found to be similar (see Ref. [23]). $\xi(t)$ increases up to time $4 \tau_{\alpha}$ and decreases at a later time. Our observations are very robust over different temperatures and density regimes. This seems to suggest that a hard-sphere-like model is probably different from those models where the particles are treated as point particles. Although these observations corroborate the previous observations, the reason for it is not immediately clear. To check the similarity in behavior of $\xi$ with time for soft- and hard-sphere-like systems, we systematically tune the softness of the interaction potential in the $3 \mathrm{dHP}$ model from $\delta=2$ to 3 . The nature of heterogeneity is found to be different. Notably, we found that $\xi(t)$ reaches a peak at $t=\tau_{\alpha}$ 
for $\delta=3$ in contrast to the scenario for $\delta=2$. Also, for the $3 \mathrm{dHP}$ model, $\xi(t) \sim \log (t)$, in agreement with Ref. [47] for hard-sphere systems. Note that the dependence for the $3 \mathrm{dR} 10$ model is very different. The mutual dependence between $\chi_{4}(t)$ and $\xi(t)$, as $\chi_{4}(t) \sim \xi(t)^{2-\eta}$, seems to have two different regimes. It is power-law-like with exponent $2-\eta \sim 4$ for all the models [4.18 (3dKA), 3.50 (3dR10), and 3.39 (3dHP)] for times up to $t \sim \tau_{\alpha}$, as shown in the insets of Fig. 5 . On the other hand, it is very different for $t>\tau_{\alpha}$. Thus it suggests that one will not be able to extract $\xi$ even approximately from the measurement of $\chi_{4}(t)$ alone.

Finally, to conclude, we have shown the presence of dynamic heterogeneity in the displacement field of particles at $\tau_{\beta}$ and highlighted the strong system size effect. We have also shown that the absolute value of the dynamic heterogeneity length may change with time but the temperature dependence of this length scale across timescales spanning from $\tau_{\beta}$ to a few $\tau_{\alpha}$ remains the same. Very surprisingly, we also found that $\xi$ obtained from the displacement-displacement correlation function remains the same in magnitude over the whole time window of study. This result is very puzzling and suggests that the usual method of extracting the correlation length from the $q \rightarrow 0$ dependence of $S_{4}(q, t)$ may not be very reliable for all time windows as it ignores the orientational correlations in the displacement of particles. We also found that the absolute value of $\xi$ reaches its maximum value at $t \sim \tau_{\alpha}$ for the $3 \mathrm{dKA}$, $3 \mathrm{dIPL}$, and $3 \mathrm{dR} 10$ models but it does so at $t \sim 4 \tau_{\alpha}$ for the $3 \mathrm{dHP}$ models. This indicates that the dynamic heterogeneity in soft-sphere models may be qualitatively different from other generic models for molecular glass formers. We believe that our findings will have important implications in colloidal and other biologically relevant glass-forming systems and may motivate experimentalists to do similar studies in these systems.

We would like to thank Chandan Dasgupta for many useful discussions during the initial phase of this work. This project is funded by intramural funds at TIFR Hyderabad from the Department of Atomic Energy (DAE). S.K. would like to acknowledge the support from Swarna Jayanti Fellowship Grants No. DST/SJF/PSA-01/2018-19 and No. SB/SFJ/2019-20/05.
[1] T. E. Angelini, E. Hannezo, X. Trepat, M. Marquez, J. J. Fredberg, and D. A. Weitz, Glass-like dynamics of collective cell migration, Proc. Natl. Acad. Sci. USA 108, 4714 (2011).

[2] T. E. Angelini, E. Hannezo, X. Trepat, J. J. Fredberg, and D. A. Weitz, Cell Migration Driven by Cooperative Substrate Deformation Patterns, Phys. Rev. Lett. 104, 168104 (2010).

[3] K. D. Nnetu, M. Knorr, J. Käs, and M. Zink, The impact of jamming on boundaries of collectively moving weak-interacting cells, New J. Phys. 14, 115012 (2012).

[4] E.-M. Schötz, M. Lanio, J. A. Talbot, and M. L. Manning, Glassy dynamics in three-dimensional embryonic tissues, J. R. Soc., Interface 10, 20130726 (2013).

[5] J. C. Shillcock, Insight or illusion? Seeing inside the cell with mesoscopic simulations, HFSP J. 2, 1 (2008).

[6] D. Bi, X. Yang, M. C. Marchetti, and M. L. Manning, MotilityDriven Glass and Jamming Transitions in Biological Tissues, Phys. Rev. X 6, 021011 (2016).

[7] D. M. Sussman, M. Paoluzzi, M. C. Marchetti, and M. L. Manning, Anomalous glassy dynamics in simple models of dense biological tissue, Europhys. Lett. 121, 36001 (2018).

[8] D. Bi, J. H. Lopez, J. M. Schwarz, and M. L. Manning, A density-independent rigidity transition in biological tissues, Nat. Phys. 11, 1074 (2015).

[9] L. Berthier and G. Biroli, Theoretical perspective on the glass transition and amorphous materials, Rev. Mod. Phys. 83, 587 (2011).

[10] E. R. Weeks, J. C. Crocker, A. C. Levitt, A. Schofield, and D. A. Weitz, Three-dimensional direct imaging of structural relaxation near the colloidal glass transition, Science 287, 627 (2000).

[11] M. D. Ediger, Spatially heterogeneous dynamics in supercooled liquids,Annu. Rev. Phys. Chem. 51, 99 (2000).

[12] S. Gokhale, R. Ganapathy, K. H. Nagamanasa, and A. K. Sood, Localized Excitations and the Morphology of Cooperatively
Rearranging Regions in a Colloidal Glass-Forming Liquid, Phys. Rev. Lett. 116, 068305 (2016).

[13] M. T. Cicerone, F. R. Blackburn, and M. D. Ediger, Anomalous diffusion of probe molecules in polystyrene: Evidence for spatially heterogeneous segmental dynamics, Macromolecules 28, 8224 (1995).

[14] L. Berthier, G. Biroli, J.-P. Bouchaud, L. Cipelletti, and W. van Saarloos, Dynamical Heterogeneities in Glasses, Colloids, and Granular Media (Oxford University Press, Oxford, UK, 2011).

[15] R. Yamamoto and A. Onuki, Kinetic heterogeneities in a highly supercooled liquid, J. Phys. Soc. Jpn. 66, 2545 (1997).

[16] A. S. Keys, A. R. Abate, S. C. Glotzer, and D. J. Durian, Measurement of growing dynamical length scales and prediction of the jamming transition in a granular material, Nat. Phys. 3, 260 (2007).

[17] A. Widmer-Cooper, P. Harrowell, and H. Fynewever, How Reproducible are Dynamic Heterogeneities in a Supercooled Liquid? Phys. Rev. Lett. 93, 135701 (2004).

[18] S. Karmakar, C. Dasgupta, and S. Sastry, Growing length scales and their relation to timescales in glass-forming liquids, Annu. Rev. Condens. Matter Phys. 5, 255 (2014).

[19] I. Tah, S. Sengupta, S. Sastry, C. Dasgupta, and S. Karmakar, Glass Transition in Supercooled Liquids with Medium-Range Crystalline Order, Phys. Rev. Lett. 121, 085703 (2018).

[20] L. Berthier, G. Biroli, J.-P. Bouchaud, L. Cipelletti, D. El Masri, D. L'Hôte, F. Ladieu, and M. Pierno, Direct experimental evidence of a growing length scale accompanying the glass transition, Science 310, 1797 (2005).

[21] D. Fragiadakis and C. M. Roland, Role of structure in the $\alpha$ and $\beta$ dynamics of a simple glass-forming liquid, Phys. Rev. E 95, 022607 (2017).

[22] S. Karmakar, C. Dasgupta, and S. Sastry, Short-Time Beta Relaxation in Glass-Forming Liquids is Cooperative in Nature, Phys. Rev. Lett. 116, 085701 (2016). 
[23] See Supplemental Material at http://link.aps.org/supplemental/ 10.1103/PhysRevResearch.2.022067 for details related to models and methods and other supporting analyses that are referred to in this Rapid Communication.

[24] W. Kob, C. Donati, S. J. Plimpton, P. H. Poole, and S. C. Glotzer, Dynamical Heterogeneities in a Supercooled LennardJones Liquid, Phys. Rev. Lett. 79, 2827 (1997).

[25] P. H. Poole, C. Donati, and S. C. Glotzer, Spatial correlations of particle displacements in a glass-forming liquid, Physica A: Stat. Mech. Appl. 261, 51 (1998).

[26] C. Donati, S. C. Glotzer, and P. H. Poole, Growing Spatial Correlations of Particle Displacements in a Simulated Liquid on Cooling Toward the Glass Transition, Phys. Rev. Lett. 82, 5064 (1999).

[27] B. Doliwa and A. Heuer, Cooperativity and spatial correlations near the glass transition: Computer simulation results for hard spheres and disks, Phys. Rev. E 61, 6898 (2000).

[28] F. Puosi and D. Leporini, Spatial displacement correlations in polymeric systems, J. Chem. Phys. 136, 164901 (2012).

[29] W. Kob and H. C. Andersen, Testing mode-coupling theory for a supercooled binary Lennard-Jones mixture I: The van Hove correlation function, Phys. Rev. E 51, 4626 (1995).

[30] S. Karmakar, E. Lerner, I. Procaccia, and J. Zylberg, Statistical physics of elastoplastic steady states in amorphous solids: Finite temperatures and strain rates, Phys. Rev. E 82, 031301 (2010).

[31] U. R. Pedersen, T. B. Schrøder, and J. C. Dyre, Repulsive Reference Potential Reproducing the Dynamics of a Liquid with Attractions, Phys. Rev. Lett. 105, 157801 (2010).

[32] C. S. O'Hern, S. A. Langer, A. J. Liu, and S. R. Nagel, Random Packings of Frictionless Particles, Phys. Rev. Lett. 88, 075507 (2002).

[33] A. J. Liu and S. R. Nagel, The jamming transition and the marginally jammed solid, Annu. Rev. Condens. Matter Phys. 1, 347 (2010).

[34] K. Kim and S. Saito, Multiple time scales hidden in heterogeneous dynamics of glass-forming liquids, Phys. Rev. E 79, 060501(R) (2009).

[35] H. Mizuno and R. Yamamoto, Dynamical heterogeneity in a highly supercooled liquid: Consistent calculations of correlation length, intensity, and lifetime, Phys. Rev. E 84, 011506 (2011).

[36] C. Bennemann, C. Donati, J. Baschnagel, and S. C. Glotzer, Growing range of correlated motion in a polymer melt on cooling towards the glass transition, Nature (London) 399, 246 (1999).

[37] E. R. Weeks, J. C. Crocker, and D. A. Weitz, Short- and long-range correlated motion observed in colloidal glasses and liquids, J. Phys.: Condens. Matter 19, 205131 (2007).

[38] S. Chakrabarty, I. Tah, S. Karmakar, and C. Dasgupta, Block Analysis for the Calculation of Dynamic and Static Length Scales in Glass-Forming Liquids, Phys. Rev. Lett. 119, 205502 (2017).

[39] C. Dasgupta, V. A. Indrani, S. Ramaswamy, and K. M. Phani, Is there a growing correlation length near the glass transition?, Europhys. Lett. 15, 307 (1991).

[40] S. Karmakar, C. Dasgupta, and S. Sastry, Growing length and time scales in glass-forming liquids, Proc. Natl. Acad. Sci. USA 106, 3675 (2009).

[41] N. Lacevic, W. F. Star, B. T. Schrøder, and C. S. Glotzer, Spatially heterogeneous dynamics investigated via a timedependent four-point density correlation function, J. Chem. Phys. 119, 7372 (2003).

[42] G. Biroli, J.-P. Bouchaud, K. Miyazaki, and D. R. Reichman, Inhomogeneous Mode-Coupling Theory and Growing Dynamic Length in Supercooled Liquids, Phys. Rev. Lett. 97, 195701 (2006).

[43] L. Berthier, G. Biroli, J.-P. Bouchaud, W. Kob, K. Miyazaki, and D. R. Reichman, Spontaneous and induced dynamic fluctuations in glass formers. I. General results and dependence on ensemble and dynamics, J. Chem. Phys. 126, 184503 (2007).

[44] L. Berthier, G. Biroli, J.-P. Bouchaud, W. Kob, K. Miyazaki, and D. R. Reichman, Spontaneous and induced dynamic correlations in glass formers. II. Model calculations and comparison to numerical simulations, J. Chem. Phys. 126, 184504 (2007).

[45] R. S. L. Stein and H. C. Andersen, Scaling Analysis of Dynamic Heterogeneity in a Supercooled Lennard-Jones Liquid, Phys. Rev. Lett. 101, 267802 (2008).

[46] C. Toninelli, M. Wyart, L. Berthier, G. Biroli, and J.-P. Bouchaud, Dynamical susceptibility of glass formers: Contrasting the predictions of theoretical scenarios, Phys. Rev. E 71, 041505 (2005).

[47] E. Flenner, M. Zhang, and G. Szamel, Analysis of a growing dynamic length scale in a glass-forming binary hard-sphere mixture, Phys. Rev. E 83, 051501 (2011). 